\title{
Processing and Visualizing the Low Earth Orbit Radio Frequency Spectrum Measurement Results From the SMOG Satellite Project
}

\author{
Donát Takács, Boldizsár Markotics, and Levente Dudás
}

\begin{abstract}
On December 6, 2019, the second and third Hungarian satellites, SMOG-P and ATL-1 (both having been developed at the Budapest University of Technology and Economics) were launched. They both had a radio frequency spectrum analyzer on board, which was used to measure for the first time the strength of radio frequency signals radiated into space by terrestrial digital TV transmitters - that can be detected in orbit around the Earth. In this paper, we present how two- and three-dimensional radiosmog maps were created from raw data received from space. The goal of this paper is to demonstrate the process of creating these maps from the raw data collected; the analysis of the results visible in these maps is beyond the scope of the present discussion.
\end{abstract}

Index Terms-DVB-T Electromagnetic Pollution Map, Educational Student Satellite, PocketQube, Radiofrequency Smog, Spectrum Monitoring.

\section{INTRODUCTION}

$\mathbf{S}$ MOG-P is a 1-PQ (PocketQube) class student satellite $(5 \times 5 \times 5 \mathrm{~cm})$, ATL- 1 is $2-\mathrm{PQ}(5 \times 5 \times 10 \mathrm{~cm})$. SMOG-P was the first and smallest operational satellite in the world during its lifetime. First, the hardware used for the measurements is presented. Then a detailed description of the data processing follows, emphasizing the solutions provided for the unique challenges posed by the special circumstances of the measurements. These include filtering out the more reliable measurement data on a statistical basis and an inverse distance weighted interpolation of the filtered data. Finally several methods for visualizing the processed data are presented, including two-dimensional cartographic projections and a threedimensional interactive web-application. https://gnd.bme.hu.

\section{HARDWARE}

The SMOG-P and ATL-1 PocketQube class satellites contain the following sub-systems (SMOG-P is in Fig. 1. left before the integration): EPS - electrical power system including solar panels, maximal power point tracker circuits, battery charger \& controller, limiter switches, step-down converters; OBC - on-board computer (micro-controller) with motion \& magnetic sensor, real-time-clock and calendar and on-board memory; COM - communication and spectrum monitoring

Boldizsár Markotics, Donát Takács are BSc and MSc university students, respectively, at Budapest University of Technology and Economics (BME), Budapest, Hungary.

Levente Dudás $\mathrm{PhD}$ as supervisor is communicational and system engineer of SMOG student satellite project at the dept. of Broadband Infocommunications and Electromagnetic Theory, faculty of Electrical Engineering and Informatics, BME. (e-mail: dudas.levente@vik.bme.hu)

DOI: 10.36244/ICJ.2021.1.3
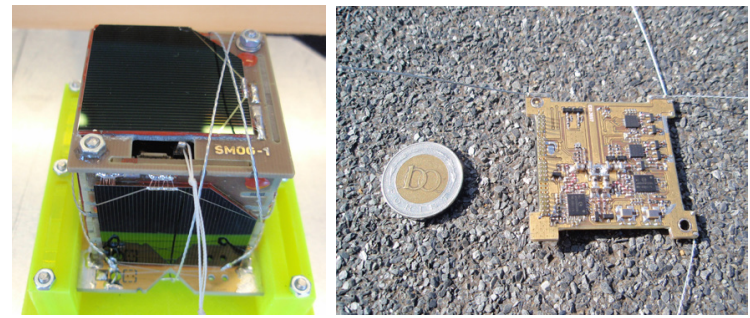

Fig. 1. The SMOG-P satellite before the integration in Glasgow (09-09-2019) - left; communication and spectrum monitoring sub-system - right

sub-system - Fig. 1 right: two independent UHF (ultra high frequency) transceiver and spectrum monitoring receiver based on Silabs ${ }^{1}$ single-chip-radio type SI1060 and SI4464. The spectrum analyzer sub-system is a conventional heterodyne radio receiver with digital intermediate frequency: carrier 119 $960 \mathrm{MHz}$; bandwidth 1.5-800 kHz; RSSI (received signal strength) range $-10 \ldots-120 \mathrm{dBm}$.

\section{DATA PROCESSING}

\section{A. Measurement data collected}

A single spectrum measurement has the following data associated with it: a UTC (Universal Time Coordinated) timestamp with a resolution of one second $(t, \mathrm{~s})$; the latitude $(\varphi, \mathrm{deg})$, longitude $(\lambda, \mathrm{deg})$ and altitude $(h, \mathrm{~km})$ coordinates calculated from the TLE (two-lines element) records and the timestamp; the measurement frequency $(f, \mathrm{~Hz})$ and the result of the measurement itself (RSSI, dBm). Thus the entire spectrum measurement dataset can be formalized as an ordered set of tuples:

$$
S=\left\{\left(t_{i}, \operatorname{RSSI}_{i}, \varphi_{i}, \lambda_{i}, h_{i}, f_{i}\right)\right\}, \quad i=1 \ldots N,
$$

where $N=19115754$ is the number of measurements performed during the two missions, namely between the 6th of December 2019 and 9th of October 2020.

\section{B. Aggregation regions}

As explained in the previous section, there is no available orientation data for the spectrum measurements, so no single measurement result can be treated as reliable in itself: it is possible that at the time of any measurement, the antenna was

${ }^{1}$ https://silabs.com 
pointing away from the Earth. This means that we had to use a statistical approach; if a sufficient number of measurements are performed above a certain region, there must be at least a few that were performed with a correct orientation, since it is known from telemetry data that the satellites were constantly rotating. If these measurements can be selected, then the real intensity of the electromagnetic field in a certain frequency band can be estimated with a reasonable confidence. It is certain that the correct measurement at a given location is that with the highest RSSI value, so it can be selected in a straightforward way. However, determining reasonable regions from which the local maxima can be selected is not straightforward: this aggregation must be defined carefully.

As seen in Figure 2, the spatial distribution of the measurements is highly inhomogeneous, due to the location of the participating ground stations and the varying enthusiasm of amateur radio operators uploading their received data. The aggregation and interpolation methods were chosen in a way that they mitigate the negative effects this distribution as much as possible.

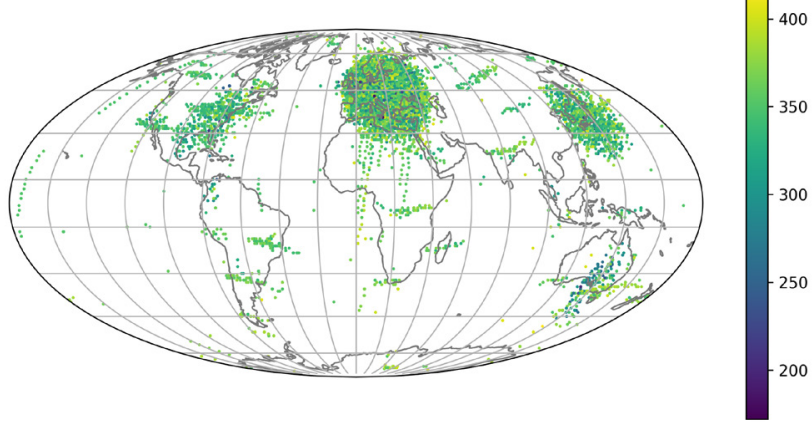

Fig. 2. The spatial distribution of the spectrum measurements performed by the satellites.

The spatial and frequency partitioning was performed by separating the set of measurements into equivalence classes. Two measurement points belong to the same spatial region if their coordinates $\left(\varphi_{i}, \lambda_{i}\right)$ and $\left(\varphi_{j}, \lambda_{j}\right)$ fulfill

$$
\begin{aligned}
& \operatorname{round}\left(\varphi_{i} / \tilde{\varphi}\right)=\operatorname{round}\left(\varphi_{j} / \tilde{\varphi}\right), \text { and } \\
& \operatorname{round}\left(\lambda_{i} / \tilde{\lambda}\right)=\operatorname{round}\left(\lambda_{j} / \tilde{\lambda}\right),
\end{aligned}
$$

where $\tilde{\varphi}$ and $\tilde{\lambda}$ are the latitudinal and longitudinal sizes of the regions, and round is the usual rounding function.

Similarly, an analogous partition of the measurement set regarding the frequency domain was performed. Since the goal is to create maps of the radio frequency transmissions for several frequency bands, simply selecting the largest measurement value in a spatial region is not a valid approach in itself. The frequency value of the measurement need to be taken into account as well. Similarly to the above, the size of a frequency region is $\tilde{f}$, and the corresponding equivalence relation is

$$
\operatorname{round}\left(f_{i} / \tilde{f}\right)=\operatorname{round}\left(f_{j} / \tilde{f}\right) \text {. }
$$

If later we would like to calculate the average RSSI value in a frequency band larger than $\tilde{f}$, the corresponding measure- ments result have to be averaged in a meaningful way, e.g. by calculating a root mean square value of the power ratios.

Equations (2)-(4) together define an equivalence relation on the set of measurements, which unambiguously partitions the measurements into distinct regions or classes. (It should be noted that this method does not yield equal-sized regions spatially due to the parametrization.) These regions will be used in a subsequent step, but first the effects of the varying altitude has to be taken into account.

\section{Reduction to mean spherical surface}

As seen in Figure 3, the altitude of measurements varies in time due to the eccentricity of the orbits and the gradually decreasing apogee. It would be of interest to create radiosmog maps for different altitudes, however the measurements available were insufficient for this. Thus the RSSI values had to be reduced to a mean middle surface; this is a perfect sphere with a mean altitude of $\bar{h}=347 \mathrm{~km}$, indicated in Figure 3 with a horizontal line.

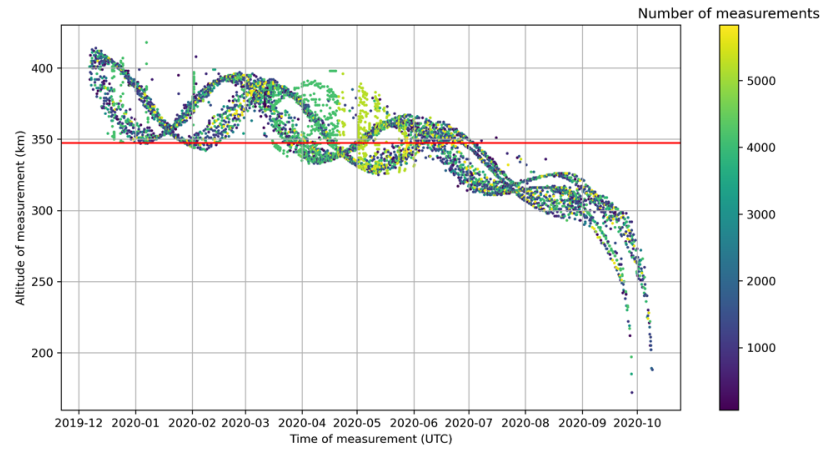

Fig. 3. The altitude and number of measurements performed by the two satellites over time.

The reduced $\operatorname{RSSI}_{i}^{\prime}$ values are calculated based on the inverse square law of transmission power:

$$
\operatorname{RSSI}_{i}^{\prime}=\rho\left(\pi\left(\operatorname{RSSI}_{i}\right) \frac{h_{i}^{2}}{\bar{h}^{2}}\right)
$$

with

$$
\rho(P)=10 \cdot \log _{10} P, \quad \pi(\mathrm{RSSI})=10^{\mathrm{RSSI} / 10}
$$

as the formulas for converting between RSSI and the corresponding power ratio values. This gives the $S^{\prime}$ set of measurements reduced to the mean surface:

$$
S^{\prime}=\left\{\left(t_{i}, \operatorname{RSSI}_{i}^{\prime}, \varphi_{i}, \lambda_{i}, f_{i}\right)\right\}, \quad i=1 \ldots N
$$

\section{Selecting the acceptable measurements}

The relations (2)-(4) can be applied to $S^{\prime}$ yielding the reduced measurement values grouped into regions. From these regions we select the intensity measurement $\operatorname{RSSI}_{i}$ with the highest value, which is regarded as a valid measurement. The parameters $\tilde{\lambda}, \tilde{\varphi}$ and $\tilde{f}$ are chosen in a way that we can be 
reasonably confident that statistically enough measurements were performed in a region for it to contain a truly valid measurement. This yields a set of measurements that is significantly smaller in size than $S$, but the results contained are more probable to be valid:

$$
\tilde{S}=\left\{\left(\operatorname{RSSI}_{i}^{\prime}, \varphi_{i}, \lambda_{i}, f_{i}\right)\right\}, \quad i=1 \ldots \tilde{N}
$$

containing $\tilde{N}=5904$ aggregated measurement points using the parameters $\tilde{\lambda}=\tilde{\varphi}=5 \mathrm{deg}$ and $\tilde{f}=50 \mathrm{MHz}$. The spatial distribution of the filtered measurement set is depicted in Figure 4.

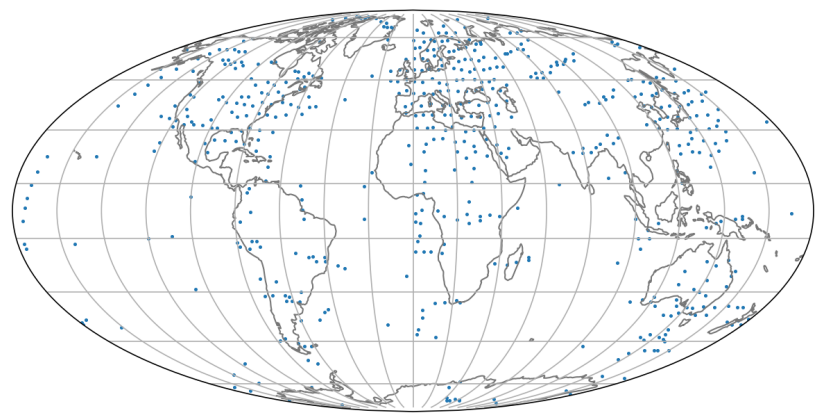

Fig. 4. The spatial distribution of the filtered measurement results on a Mollweide projection.

\section{E. Interpolation}

Since we regard the measurements in $\tilde{S}$ as valid, it is meaningful to interpolate them to get a sense of the electromagnetic field around the whole globe. The interpolation has to be performed over the mean spherical surface, so it has to take into account the two main features of the spherical geometry: its unboundedness (i.e. interpolation must be performed over the date line) and its curvature (i.e. the straight lines are the geodesics). To achieve this efficiently, we used a Python interface [1] to the established STRIPACK [2] and SSRFPACK [3] libraries used for performing calculations over triangular meshes. The mesh used was an icosahedral mesh (see Figure 5) of order 7 with the vertices and face centers as interpolation points.
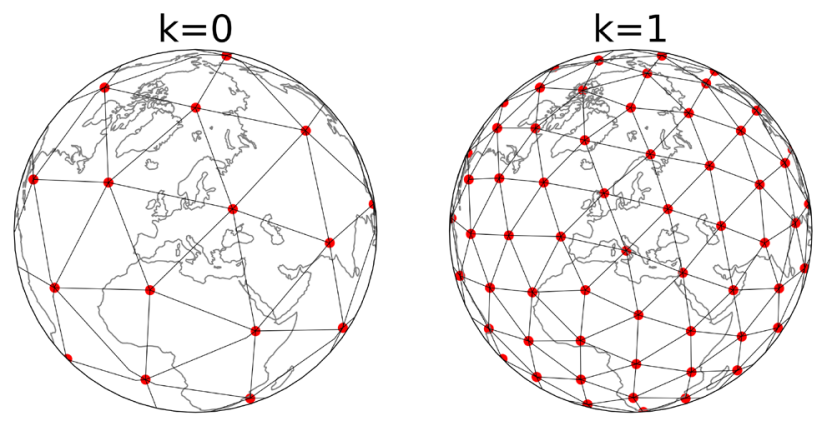

Fig. 5. The first two orders of refinement on the icosahedral mesh. The mesh used for the interpolation was of order $k=7$, which is too dense to be illustrated on a global scale.
There several methods used for interpolating RSSI measurements, such as spline interpolation, distance weighting and kriging [4] [5]. We used inverse distance weighting (IDW) of the power ratio values for the interpolation based on several reasons: this method performed well in a similar task [4]; the main drawback of the IDW method, i.e. the difficulty of determining a proper exponent of the distance $d$ to be used for the weights $w$ does not exist here, since the wave propagation in space can be considered ideal, thus $w \propto d^{-2}$; finally, of the three methods the IDW is the most tolerant to data points with measurement error, which can occur due to the nature of the dataset.

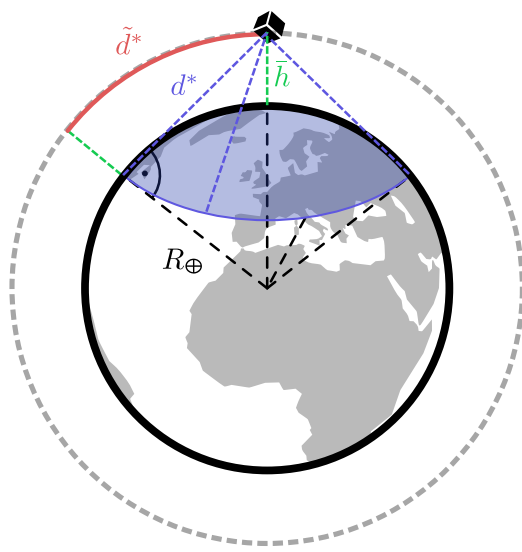

Fig. 6. A diagram illustrating the horizon of the satellite performing the measurements.

As illustrated in Figure 6, the points to be considered for the interpolation around a given satellite position are those that are above the areas on Earth visible from the satellite, i.e. within its horizon. This is simply due to the fact that radio transmitters below the horizon have no effect on the measurements performed at the given location, excluding atmospheric propagation effects. The two distances needed for performing an IDW interpolation using this method is the Euclidean distance to the horizon $d^{*}$ and the corresponding geodesic distance $\tilde{d}^{*}$ on the mean spherical surface, which can be calculated using elementary geometry:

$$
\begin{gathered}
d^{*}=\sqrt{\left(R_{\oplus}+\bar{h}\right)^{2}-R_{\oplus}^{2}}, \\
\tilde{d}^{*}=\left(R_{\oplus}+\bar{h}\right) \arcsin \left(\frac{d^{*}}{R_{\oplus}+\bar{h}}\right),
\end{gathered}
$$

with $R_{\oplus}$ denoting the mean radius of Earth. The interpolation function is thus:

$$
\operatorname{RSSI}\left(P_{0}\right)=\rho\left(\frac{\sum_{i} \pi\left(\overline{\operatorname{RSSI}}_{i}\right) w\left(P_{0}, P_{i}\right)}{\sum_{i} w\left(P_{0}, P_{i}\right)}\right), \text { if } \tilde{d}\left(P_{0}, P_{i}\right)<\tilde{d}^{*},
$$


with weights

$$
w\left(P_{0}, P_{i}\right)=\frac{1}{d\left(P_{0}, P_{i}\right)^{2}},
$$

where $d\left(P_{i}, P_{j}\right)$ denotes the Euclidean and $\tilde{d}\left(P_{i}, P_{j}\right)$ the geodesic distance between the two points. The point $P_{0}$ can be an arbitrary point on the mesh, while $P_{i}$ is a measurement point.

\section{VisUALIZATION}

With the filtered, reduced measurements in $\tilde{S}$ and the interpolation defined by (11)-(12), several approaches for visualizing the resulting radiosmog maps were used. These include 2D map projections, and a 3D interactive web-application. There is no single best visualization of the data, all have their advantages and disadvantages. All of the results presented here show the power-averaged results over the frequency band from $119 \mathrm{MHz}$ to $960 \mathrm{MHz}$.

\section{A. Map projections}

Two-dimensional map projections are used traditionally for geospatial datasets. The choice of the projection method is always a trade-off; here the Robinson projection method was used for easy interpretation of the results. All map projections were created using the open-source Python library Cartopy [6]. For these projections, a Plate Carree rectangular mesh had to be used, which is an additional interpolation on the icosahedral mesh points. The rectangular mesh was of order 6 , which was chosen to be similar in resolution to that of the icosahedral mesh. Since the resolution of these meshes is sufficiently high, a linear interpolation on a local tangent planar surface was used. The two meshes are illustrated in Figure 7.

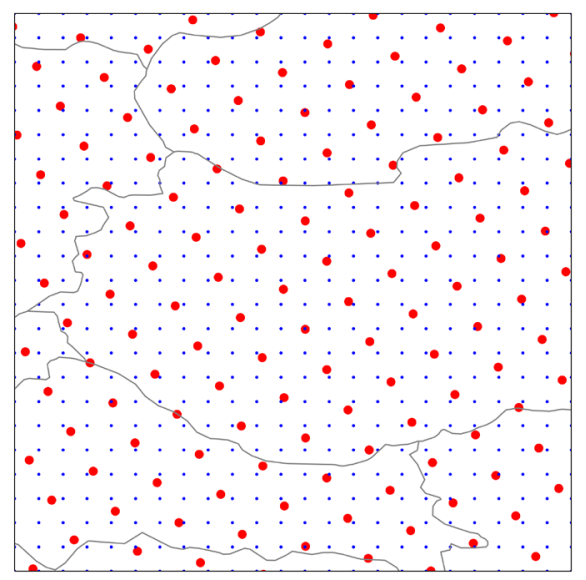

Fig. 7. A small section of the points in the two interpolation meshes (above Hungary for scale): icosahedral mesh of order 7 (red) and Plate Carree rectangular mesh of order 6 (blue).
1) Voronoi-cell RSSI map: If we would like to visualize the unaltered, but filtered measurement results (that is, $\tilde{S}$ ), a Voronoi-cell based visualization can be used. This shows the spatial regions in which a measurement point is the nearest, and gives a sense of the distribution of values. This map is shown in Figure 8. It is clearly visible here that the intensity of received signals is higher over the more densely populated regions, as expected, but the distribution of the results is not continous, which is not realistic.

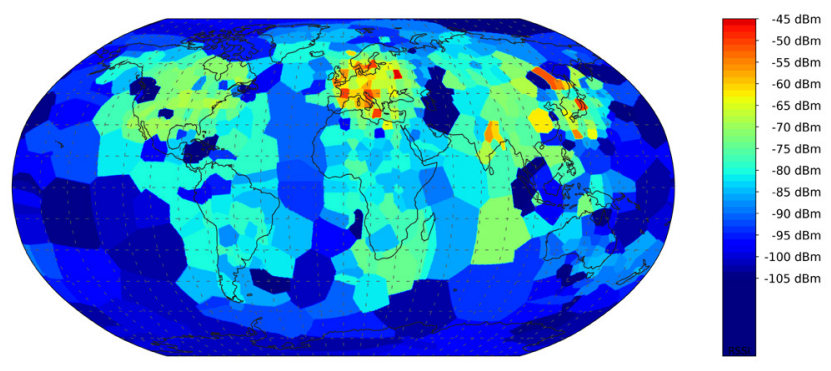

Fig. 8. The Voronoi cell map of the measurement results without interpolation.

2) Interpolated, continuous distribution RSSI map: Using the method described in subsection III-E, a continuously interpolated RSSI map can be created, which is shown in Figure 9. This interpolation takes into account the fact that the real distribution of the electromagnetic field is continous.

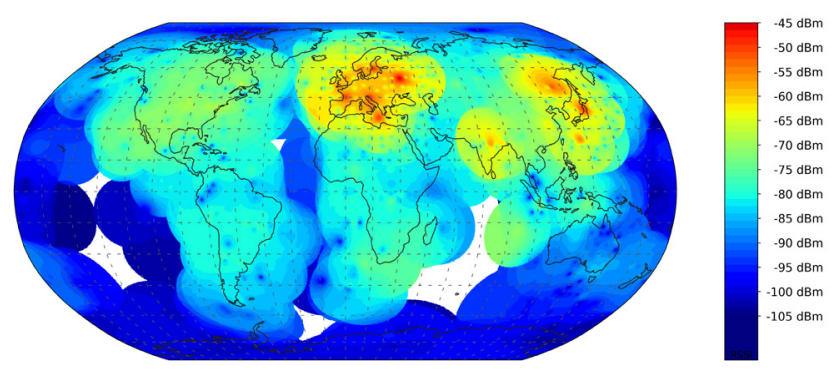

Fig. 9. The map created from IDW interpolated, filtered measurements results, using the Robinson-projection.

There several visible artifacts in this map. Due to the limiting nature of the horizon in the interpolating method, some areas (indicated in white) have no RSSI value associated: there is no valid measurement available within the horizon of these areas. In some other areas (e.g. above India), the border of the horizon-circles is sharply visible. Furthermore, in some other points (e.g. above South America) some outlier low values can be noticed. These indicate that the measurement result at those points, though treated as valid, is probably invalid: the IDW interpolation suggests that the nearby field is much stronger based on the surrounding measurement results.

3) Gauss-smoothed RSSI map: If we would like to get a qualitative sense of the entire global distribution, a smoothing of the power ratio values using a Gaussian kernel can be performed. This of course distorts the absolute value of the measurement results, thus this can only be used to estimate the overall shape and trends of the true spatial distribution of the 
radio frequency pollution from Earth reaching the satellites. The smoothed, interpolated map is shown in Figure 10.

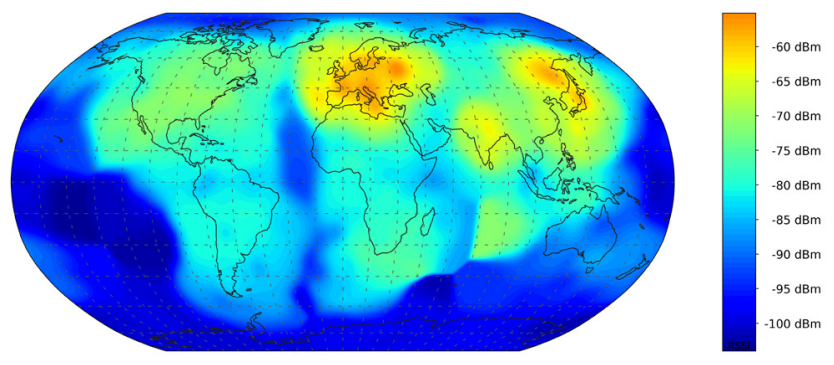

Fig. 10. The map of interpolated results of the measurement results with Gaussian smoothing.

\section{B. Interactive $3 D$ web-application}

Further, the goal was to display the now filtered, interpolated data in an effective, visually appealing way.

This was achieved using a web application based on JavaScript technology. The webGL-based library THREE.js was used for the display of the objects, because it is a lightweight and user friendly implementation of the webGL technology.

The application can display the processed data set in various ways (discrete measurement points, continuous interpolated distribution, contour lines, etc.). The page is interactive, so the user can filter the results by frequency as well as is able to influence certain properties of the displayed objects, such as their movement or the used textures on them to achieve the required user experience.

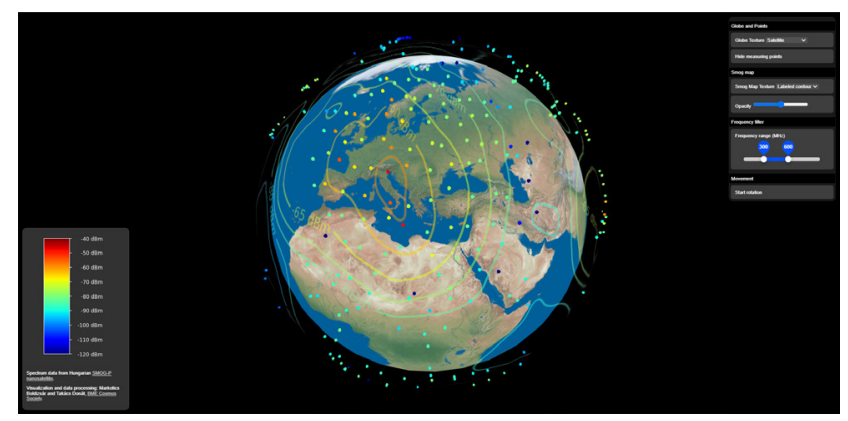

Fig. 11. A screenshot of the 3D visualization web application.

The completed web application can be viewed by anyone at https://gnd.bme.hu/mb/site/ at the time of this publication.

\section{Spectrum Measurement Results}

In the previous sections we mainly focused on the spatial distribution and magnitude visualization of the measurement data. However, the measurements also span a significant frequency range, over which the measurement results highly vary based on the measurement locations. A few examples of these spectrum measurements are shown in this section. The timestamp and the RBW (Resolution Bandwidth) parameter of the spectrum measurements are also included in the figures.
Based on the TLE (two-lines element set - Keplerian data) and the timestamp of the measurement, the actual position of the satellite can be visualized with GPredict ${ }^{2}$. These maps are also presented for reference.

Due to their altitude (365 km LEO (Low Earth Orbit)), the satellites can only receive radio signals within a ca. 4000 $\mathrm{km}$ diameter horizon (per Figure 6) - yellow circle in maps, red line shows the trajectory of the satellite. The measured spectrum is corrected with the antenna factor of the spectrum measurement system (raw RSSI data downloaded from the satellite).

\section{A. Pacific Ocean}

Over the Pacific Ocean, the noise power level of the measurement system can be detected with $384 \mathrm{kHz}$ resolution bandwidth (RBW). Inside this area, there is no TV watcher, this means: there is no DVB-T (Digital Video Broadcasting - Terrestrial) transmitter, hence there is no RF (Radio frequency) pollution: Fig. 12 - 13. In conclusion the spectrum measurement system is working.

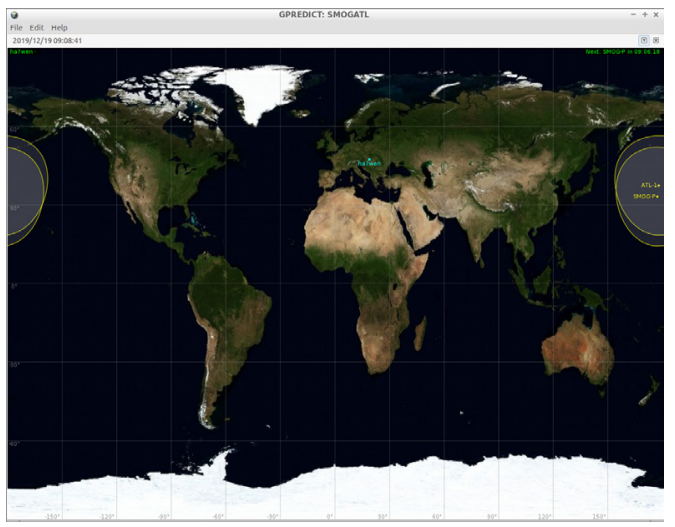

Fig. 12. Satellites over Pacific Ocean.

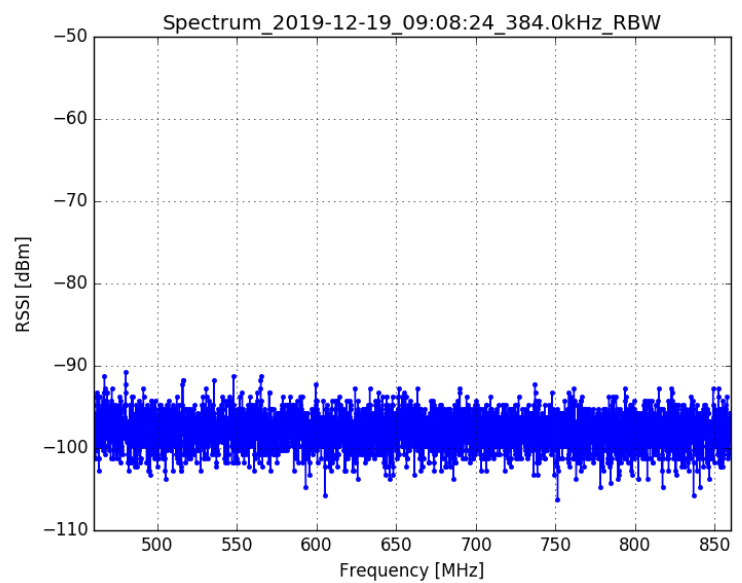

Fig. 13. Measured spectrum over Pacific Ocean on 2019. 12. 19. with 384 kHz RBW.

${ }^{2}$ http://gpredict.oz9aec.net/ 
Processing and Visualizing the Low Earth Orbit Radio Frequency Spectrum Measurement Results From the SMOG Satellite Project

\section{B. Europe}

Over Europe, $-60 \quad \ldots-80 \mathrm{dBm}$ signal level of DVB-T transmitters and between 790 and $820 \mathrm{MHz}$ with $-70 \mathrm{dBm}$ power level 5G mobile system can be measured: Fig. $14-15$. The whole lower DVB-T sub-band is continuously filled with signal of TV transmitters. The signal levels of 5G and DVB-T can be measured with almost the same signal level.

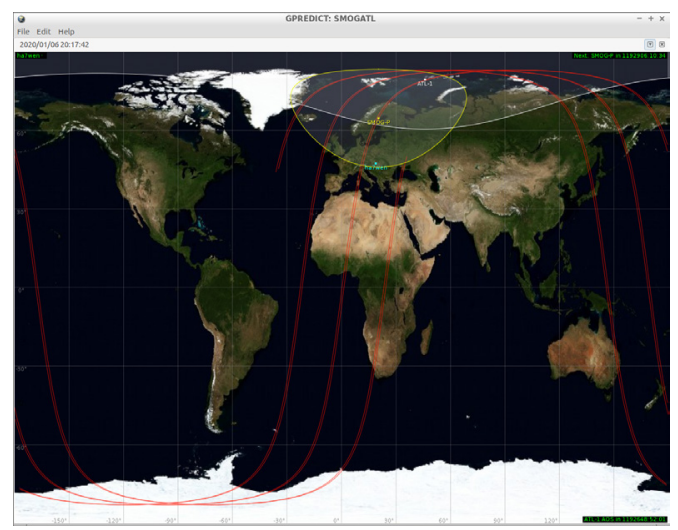

Fig. 14. Satellites over Europe.

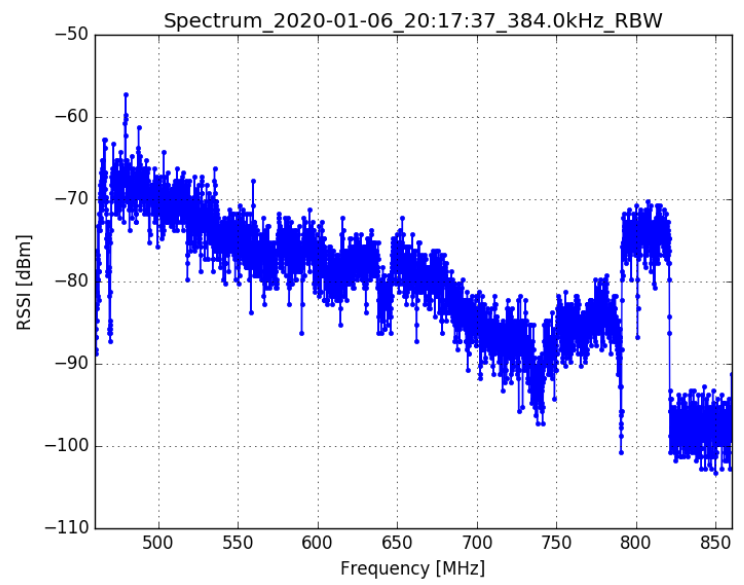

Fig. 15. Measured spectrum over Europe on 2020. 01. 06. with $384 \mathrm{kHz}$ RBW.

\section{Europe-Asia}

Between Europe and Asia, only DVB-T transmitters can be measured: Fig. 16 - 17, there is no $5 \mathrm{G}$ signal.

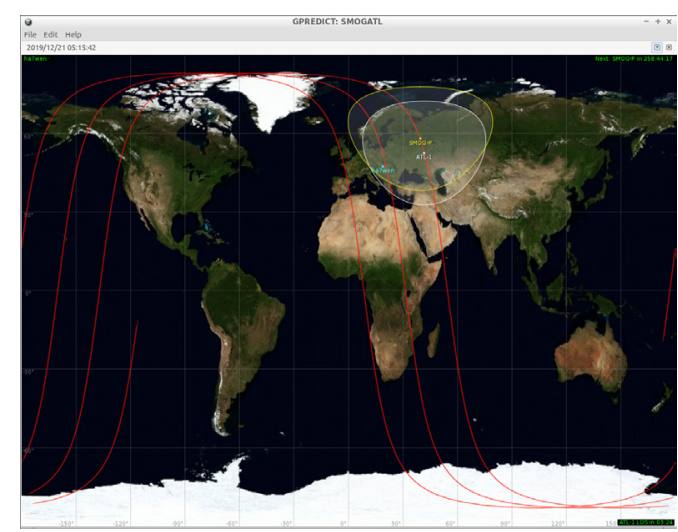

Fig. 16. Satellites over Eur-Asia.

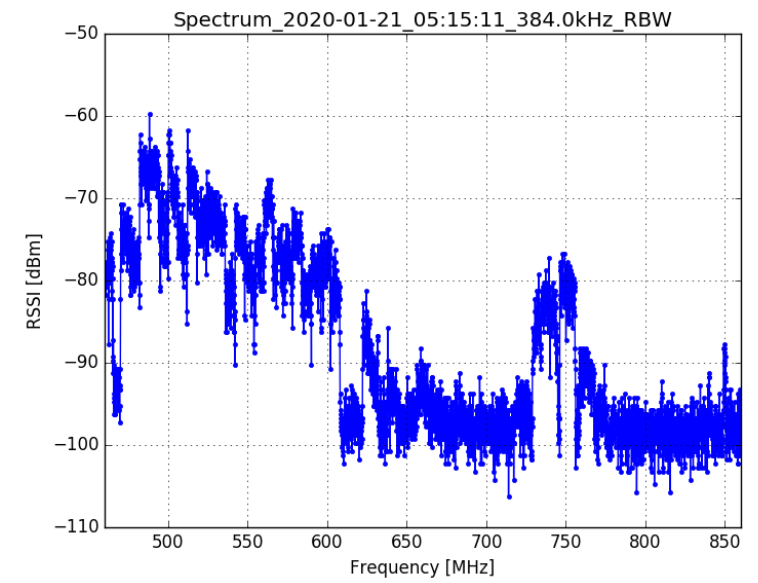

Fig. 17. Measured spectrum over Eur-Asia on 2020. 01. 21. with $384 \mathrm{kHz}$ RBW.

\section{North-America}

Over America, high-power-level of DVB-T band pollution can be measured: Fig. 18 - 19, 480-610, 625-675, 725-775 $\mathrm{MHz}$ sub-bands are filled up with TV signals, GSM band communication is visible over $850 \mathrm{MHz}$.

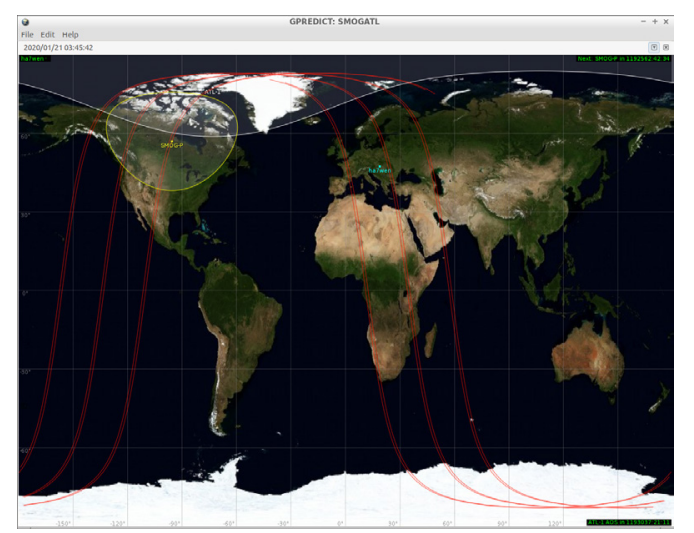

Fig. 18. Satellites over North-America. 


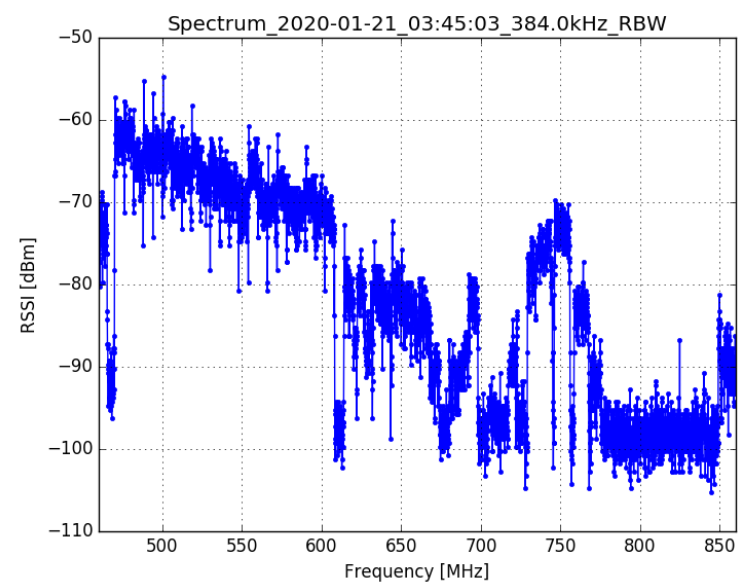

Fig. 19. Measured spectrum over North-America on 2020. 01. 21. with 384 kHz RBW.

\section{E. Central-America}

Over Central-America, there is lower power level of DVB-T band pollution detected, because of the longer range between the satellite and the DVB-T transmitters and the low-elevation radiation angle of the TV transmitters: Fig. $20-21$ - the TV transmitters radiate its RF signal to lower elevation angle ranges toward to the TV watcher (on the Earth surface).

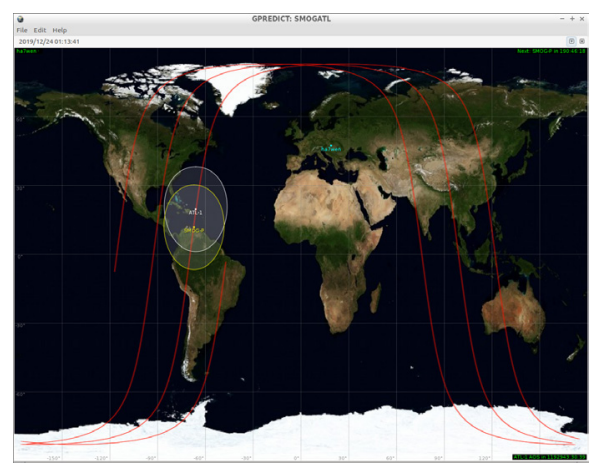

Fig. 20. Satellites over Central-America.

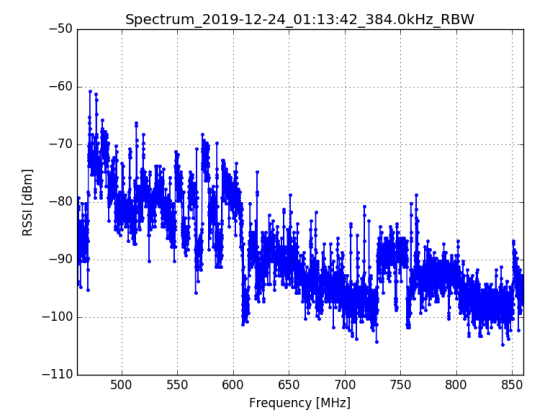

Fig. 21. Measured spectrum over Central-America on 2019. 12. 24. with 384 kHz RBW.

\section{F. Arctic and European RF pollution comparison}

Arctic and over Europe measured spectrum can be seen in Fig. 22 with the same scale: blue - European region, grey Arctic. The differences between only the noise power and the RF pollution level caused by TV transmitters is highly visible.

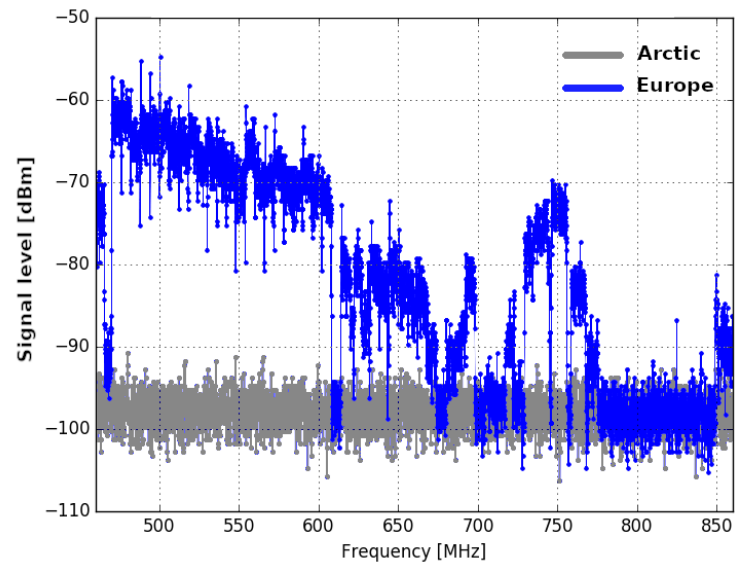

Fig. 22. Spectrum comparison between the Artic and Europe.

SMOG-P and ATL-1 mainly focused on the DVB-T band, but measured not only DVB-T: radiated RF signal of 5G and GSM band mobile phone system can be identified on the spectrum figures.

\section{CONCLUSION}

According to the presented measurement results and its visualization, the level of electromagnetic pollution caused by human beings on the digital video broadcasting terrestrial (DVB-T) frequency band had been measured: there is a huge amount of radiated power on low-Earth-orbit (LEO), that heats the space and disturbs the communication system of LEO satellites.

\section{ACKNOWLEDGMENTS}

The authors would like to thank the engineers that have worked on the SMOG-P and ATL-1 satellites, having made this analysis possible through their efforts and technical expertise. We are especially thankful for Dr. András Gschwindt for his overarching vision and for bringing us together for this work, as well as Emil Viktor Hödl for his patient answers and continuous support.

\section{Author contributions}

D.T.: implementation of filtering, idea for and implementation of interpolation method, mean surface reduction and map projections, manuscript text and figures; B.M.: idea for frequency selection, concept and implementation of $3 \mathrm{D}$ visualization, manuscript text; L.D.: spectrum analyzer hardware, spectrum measurement figures and interpretations, idea for filtering approach, manuscript text. All authors have read and agreed to the published version of the manuscript. 


\section{REFERENCES}

[1] L. Moresi and B. Mather, "Stripy: A Python module for (constrained) triangulation in Cartesian coordinates and on a sphere." Journal of Open Source Software, vol. 4, no. 38, p. 1410, Jun. 2019. [Online]. Available: http://joss.theoj.org/papers/10.21105/joss.01410

[2] R. J. Renka, “Algorithm 772: STRIPACK: Delaunay triangulation and Voronoi diagram on the surface of a sphere," ACM Transactions on Mathematical Software, vol. 23, no. 3, pp. 416-434, Sep. 1997. [Online]. Available: DoI: 10.1145/275323.275329

[3] - - "Algorithm 773: SSRFPACK: interpolation of scattered data on the surface of a sphere with a surface under tension," $A C M$ Transactions on Mathematical Software, vol. 23, no. 3, pp. 435-442, Sep. 1997. [Online]. Available: Dor: 10.1145/275323.275330

[4] M. A. Azpurua and K. D. Ramos, "A COMPARISON OF SPATIAL INTERPOLATION METHODS FOR ESTIMATION OF AVERAGE ELECTROMAGNETIC FIELD MAGNITUDE," Progress In Electromagnetics Research M, vol. 14, pp. 135-145, 2010. [Online]. Available: http://www.jpier.org/PIERM/pier.php?paper=10083103

[5] B. Li, Y. Wang, H. Lee, A. Dempster, and C. Rizos, "Method for yielding a database of location fingerprints in WLAN," IEE Proceedings - Communications, vol. 152, no. 5, p. 580, 2005. [Online]. Available: https://digital-library.theiet.org/content/journals/10.1049/ ip-com_20050078

[6] M. Office, Cartopy: a cartographic python library with a matplotlib interface, Exeter, Devon, 2010. [Online]. Available: https://scitools. org.uk/cartopy

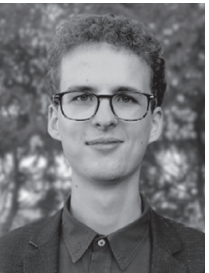

Donát Takács has received the BSc degree in mechatronical engineering at the Budapest University of Technology and Economics (BME) in 2020. He is currently pursuing the MSc in mechanical engineering modelling at BME, with a major in solid mechanics and a minor in fluid mechanics.

His research interests include general mathematical and computational modelling of various engineering phenonena, new methods in finite element analysis (such as isogeometric analysis) and failure prediction of composite materials. He is a founding member, and currently the president of the BME Cosmos Society.

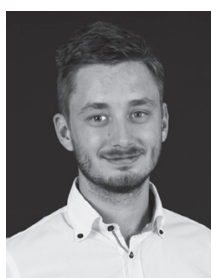

Boldizsár Markotics has ongoing BSc studies at the Budapest University of Technology and Economics (BME) as an electrical engineer in the field of High Frequency System Techniques.

Currently working on a project in Radar Research Group regarding the Doppler effect. His intrests include various radar technologies, programming languages, the theory of electromagnetic fields and lately web development. He is currently the vice president of the BME Cosmos Society.

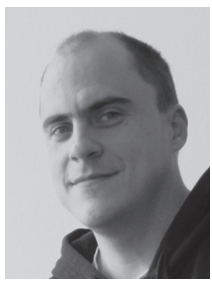

Levente Dudás has received the MSc degree in 2007 in electrical engineering, $\mathrm{PhD}$ in 2018 - Radar and Satellite Applications of Radio and Antenna Systems - at the Budapest University of Technology and Economics (BME). He is currently senior lecturer at the Department of Broadband Infocommunications and Electromagnetic Theory at BME, working as electrical engineer in Microwave Remote Sensing Laboratory, president of Radio Club of BME.

His research interests include microwave remote sensing (RADAR): active and passive radar; CubeSat and PocketQube type satellite development; analog RF hardware and antenna design; automated and remote controlled satellite control station development; Software Defined Radio based signal processing for satellite ans radar applications. 